\title{
Efecto nutrimental en floración y envero en vid para vino en la región serrana de Sonora
}

\author{
Martínez Díaz Gerardo ${ }^{1 *}$, Miranda Blanco José Luís ${ }^{1}$, Vieira de Figueiredo Fernando Afonso ${ }^{1}$, \\ Jiménez García Emílio ${ }^{1}$
}

${ }^{1}$ Campo Experimental de la Costa de Hermosillo-INIFAP-CIRNO.

\begin{abstract}
Resumen
El cultivo de vid para vino en la región de Cananea, noreste de Sonora, es reciente y se desconoce el contenido de los niveles nutrimentales del mismo. Se determinó la concentración de macro y micronutrientes durante las etapas de floración y envero, en pecíolo y lámina de la hoja. Las muestras fueron colectadas en los 16 cultivares de vid que se encuentran injertados sobre seis diferentes portainjertos y que fueron plantados en la primavera del 2011, en el Ejido Zaragoza en el Municipio de Cananea, Sonora, México. Los resultados muestran que la concentración de los nutrientes $\mathrm{NO}_{3}{ }^{-1}, \mathrm{HPO}_{4}^{-2}, \mathrm{~K}^{+}, \mathrm{Ca}^{++}, \mathrm{Mg}^{+}+, \mathrm{Zn}^{++}, \mathrm{Na}^{+}$fue estadísticamente $(\mathrm{p}<0.05)$ mayor en el tejido del pecíolo que en la hoja, en la época de floración, En contraste, la concentración de $\mathrm{BO}_{4^{\prime}} \mathrm{Fe}^{++}$y $\mathrm{Mn}^{++}$fue mayor ( $\mathrm{p}>0.05$ ) en el tejido de la hoja. La concentración de $\mathrm{Cu}^{++}$y $\mathrm{SO}_{4}^{-2}$ no mostró diferencias ( $\left.\mathrm{p}>0.05\right)$ entre peciolo y hoja. La concentración de los elementos $\mathrm{NO}_{3}^{-1}, \mathrm{HPO}_{4}^{-2}, \mathrm{~K}^{++}, \mathrm{Mg}^{++}, \mathrm{Mn}^{++}, \mathrm{BO}_{4}^{-}$en peciolos fue mayor $(\mathrm{p}>0.05)$ durante la floración que durante el envero, mientras que en el resto de los elementos se registró lo contrario.
\end{abstract}

Palabras clave: Acronutriente, micronutriente, diagnóstico nutrimental, condición desértica.

\begin{abstract}
Wine grape crop in the region of Cananea, northeastern Sonora, has been recently established, for which the content of nutritional levels is unknown. In order to know then, the concentration of macro and micronutrients was determined during the stages of flowering and veraison, in petiole and leaf blade tissue. The samples of tissues were collected in the 16 vine cultivars, which are grafted on six different rootstocks that were planted in the spring of 2011, in the area of Ejido Zaragoza in the Municipality of Cananea, Sonora, Mexico. Results show that the concentration of $\mathrm{NO}_{3}^{-1}, \mathrm{HPO}_{4}^{-2}, \mathrm{~K}^{+}, \mathrm{Ca}^{++}, \mathrm{Mg}^{++}, \mathrm{Zn}^{++}, \mathrm{Na}{ }^{+}$was statistically $(\mathrm{p}<0.05)$ higher in petiole than in the leaf blade, at the time of flowering. In contrast, concentration of $\mathrm{BO}_{4}^{-}, \mathrm{Fe}^{++}$and $\mathrm{Mn}^{++}$was greater ( $\mathrm{p}>0.05$ ) in the leaf tissue. In addition, for concentration of $\mathrm{Cu}++$ and $\mathrm{SO}_{4}^{-2}$ no differences were shown ( $\left.\mathrm{p}>0.05\right)$ between petiole and leaf. Finally, concentration of $\mathrm{NO}_{3}{ }^{-1}$, $\mathrm{HPO}_{4}^{-2}, \mathrm{~K}^{++}, \mathrm{Mg}^{++}, \mathrm{Mn}^{++}, \mathrm{BO}_{4}$ in petioles was greater $(\mathrm{p}>0.05)$ during flowering than in veraison, while with the concentration of the rest of elements was inverse $(\mathrm{p}<0.05)$.
\end{abstract}

Nutrient effect at flowering and veraison in wine grapes in highland region of Sonora

Keywords: Macronutrient, micronutrient, nutrient assessment, desert condition. 


\section{INTRODUCCIÓN}

El cultivo de vid para vino (Vitis vinífera L.) en la región serrana de Cananea, Sonora, es reciente y se tienen establecidas plantaciones piloto a nivel experimental y semicomercial con diferentes cultivares. El propósito de esta plantación es generar tecnología que soporte el establecimiento de una industria vitivinícola en esta localidad, ya que es un área que posee las condiciones agroecológicas propicias para el desarrollo de cultivares de vid para producción de uva para tales propósitos (Martínez y Moreno, 2016). El conocimiento de la condición nutrimental de las plantas de vid en esta nueva área agroecológica es de gran relevancia, debido a que hace posible determinar la concentración real de los macro y micronutrientes. Esta información hará posible suministrar los nutrientes requeridos por las plantas de vid, mediante aplicaciones al suelo o al follaje a fin de lograr obtener el rendimiento y calidad óptimos de las uvas para vinificación (Christensen, 1984; Vallone, 2002; Davenport y col., 2003; Palma, 2006).

El estado nutrimental de plantaciones de vid se determina comúnmente mediante el análisis químico de tejidos de peciolos o de lámina de hojas. Y la colecta de las muestras de dichos tejidos normalmente se efectúa en la fase de plena floración o durante el envero (Robinson y col., 1978; Christensen, 1984; Whiting, 1990; Nagarajah, 1999; Romero y col., 2005; Davenport y col., 2011). En la actualidad aún existe desacuerdo entre investigadores respecto a la etapa fenológica y sección de la hoja más adecuada para efectuar el muestreo para determinar el estado nutrimental de las plantas de vid (Christensen, 1984; Fallahi y col., 2005; Romero y col., 2013). La información disponible indica que la etapa fenológica y sección de hoja que se deben muestrear para determinar el contenido nutrimental de plantas de vid dependen del tipo de nutriente a considerar cuando se pretende tener un conocimiento general del estado nutrimental de la planta. Algunos investigadores proponen que la cuantificación de los niveles nutrimentales se realice en tejidos de peciolos y cuando la parra está en plena floración (Christensen, 1984; Nagarajah, 1999; Romero y col., 2005).

En lo que respecta a la concentración de nutrientes, para el caso de los cultivares de vid para uva de mesa y que se cultivan bajo las condiciones del desierto de Sonora, se ha observado que los niveles de nutrimentos de las parras de plantaciones en estas localidades son muy diferentes a los que se reportan de plantaciones bajo condiciones de clima mediterráneo (Márquez y col., 2004; Huvely y col., 2014). Las plantaciones de vid en condiciones de clima mediterráneo están expuestas a las precipitaciones que ocurren principalmente durante el invierno, a condiciones de temperatura muy favorables y sobre todo una buena acumulación de frío invernal, en contraste con las que se desarrollan en el desierto de Sonora. En esta zona, las precipitaciones se presentan en una mayor proporción durante el verano, con temperaturas altas durante el ciclo de crecimiento y una acumulación de frío invernal deficiente. Además, los suelos en estas áreas, en comparación con los de clima mediterráneo, son muy diferentes en sus características fisicoquímicas, ya que los suelos de las áreas desérticas tienen un origen de roca caliza principalmente (Yaalon, 1997; Márquez y col., 2004 ; Peel y col., 2007).

En las diferentes regiones productoras de vid para vino en condiciones de clima mediterráneo, se ha encontrado que el contenido nutrimental es similar entre ellas, con mínimas variaciones por la influencia del clima, suelos y niveles de humedad relativa, (Kilby, 1999; Vallone, 2002; Romero y col., 2005; Palma, 2006; Davenport y col., 2011; Huvely y col., 2014). Sin embargo, en las regiones con condiciones de clima semidesértico, como la de Sonora, se desconoce la condición nutrimental de las variedades de vid para producción de vino. La reciente introducción y plantación de cultivares de vid para vino en estas regiones presenta la oportunidad para determinar el estado nutrimental de dichos cultivares bajo estas condiciones de desarrollo. Estos resultados permitirían elaborar fórmulas de nutrición aplicables a zonas desérticas de Sonora, México y diferentes a aquellas de climas mediterráneos, tal como ocurre en las uvas para mesa. La aplicación de fórmulas nutrimentales específicas para esta zona desértica es un objetivo de esa investigación.

\section{MATERIALES Y MÉTODOS}

El presente estudio se llevó a cabo en la plantación piloto de cultivares de vid para vino, establecida en terrenos del ejido Zaragoza ubicado en el municipio de Cananea; Sonora, con coordenadas $31^{\circ}$ 05' $12.32^{\prime \prime} \mathrm{N}$ y $110^{\circ} 04^{\prime} 54.42^{\prime \prime} \mathrm{O}$ y con elevación de 1554 msnm. El clima es semiseco templado $\mathrm{BS}_{1} \mathrm{~K}$ (García, 1988), con variaciones de temperatura máximas de 14.8 a $37.7^{\circ} \mathrm{C}$ y mínimas de -1.21 a $21.9{ }^{\circ} \mathrm{C}$. El suelo, de textura arcillo arenosa con baja compactación, presentó un $\mathrm{pH}$ de 7.0 a nivel superficial y ligeramente básico en profundidades mayores. El contenido de materia orgánica fue de $1.3 \%$, con un contenido de nitrógeno con grado de evolución medio $8(\mathrm{C} / \mathrm{N}=9)$, considerado como moderado. La relación Capacidad de Intercambio Catiónico (CIC) presentó valores de 5.6 a $9.0 \mathrm{Cmol}+/ \mathrm{Kg}$, con un equilibrio mineral de dominancia del $\mathrm{Mg}$ sobre el $\mathrm{K}$.

El lote de la plantación consistió de 16 cultivares injertados sobre seis diferentes portainjertos, con material procedente de Mercier, Francia, el cual fue establecido en la primavera del año 2011. Para determinar el efecto de la etapa fenológica en la concentración nutrimental de los cultivares 
en evaluación se realizaron muestreos de tejidos en etapa de floración y envero. En el caso de la etapa de envero, el muestreo se efectuó el 20 de julio del 2012, fecha en que se colectaron 120 muestras de peciolos. Para etapa de floración el muestreo se efectuó el 24 de mayo de 2014. Se colectaron 120 muestras de peciolos y 120 láminas de hojas en cada uno de los diferentes cultivares. Los cultivares de vid para vino muestreados fueron: Grenache Noir, Malbec, Tempranillo, Petit Verdot, Syrah, Touriga Nacional, Sauvignon Blanc, Cabernet Sauvignon, Marsanne, Mourvèdre, Chardonnay, Marselan, Verdejo, Macabeu, Merlot y Roussanne.

Una vez colectadas, las muestras de tejido fueron colocadas en bolsas de papel Kraft 65, secadas al ambiente y remitidas posteriormente a un laboratorio comercial para su análisis. Para efectuar el análisis, las muestras de los peciolos y hojas fueron lavadas primeramente con agua corriente y posteriormente, en tres ocasiones, con agua destilada y secadas en una estufa eléctrica a $100{ }^{\circ} \mathrm{C}$. Los elementos tipo catiónicos como $\mathrm{K}^{+}, \mathrm{Ca}^{++}, \mathrm{Mg}^{++}, \mathrm{Na}^{+}, \mathrm{Cu}^{++}, \mathrm{Zn}^{++}$, $\mathrm{Mn}^{++}$y $\mathrm{Fe}^{++}$fueron determinados mediante la técnica de espectrometría de absorción atómica y los tipos aniónicos, $\mathrm{SO}_{4}{ }^{2}, \mathrm{BO}_{4}, \mathrm{NO}_{3}{ }^{-1}$ y $\mathrm{HPO}_{4}{ }^{-2}$, mediante la técnica de colorimetría (Reuters y Robinson, 1977; Anónimo, 1996).

Los datos de concentración de los nutrientes en los cultivares considerados, tanto en los tejidos de peciolos como en lámina de hoja en las etapas de floración y envero, fueron concentrados, promediados y sometidos a la prueba de diferencia mínima significativa $(\mathrm{T} \leq \mathrm{t})$ utilizando el paquete estadístico SAS. Así mismo, se calculó el coeficiente de variación para cada elemento en cada etapa fenológica y tejido considerado.

\section{RESULTADOS Y DISCUSIÓN}

Los datos promedio de las concentraciones de nutrientes para macro y micronutrientes en los dos tejidos y dos etapas fenológicas de los cultivares de vid, se muestran en las tablas 1 y 3. Al realizar la comparación de las concentraciones de los macro y microelementos, en los tejidos de peciolos contra lamina de las hojas al momento de la floración (Tabla 1), la concentración en peciolos fue estadística mayor $(p<0.01)$ que en la lámina de las hojas, con excepción del $\mathrm{Cu}^{++}$y $\mathrm{SO}_{4}^{-2}$ que no presentaron diferencia estadísticamente significativa. Estos resultados concuerdan con los reportado por los estudios de Romero y col. (2013).

\section{Concentración de nutrientes en peciolos y hojas durante floración}

En la Tabla 1 se observa la concentración de los nutrientes en los peciolos y láminas de la hoja, al momento de la floración. Los macro y micro nutrientes analizados presentan concentraciones promedio superiores a lo considerado como óptimo en cultivares de vid para vino, con excepción del $\mathrm{Mg}^{++}$, según los estándares reportados en otras regiones y países (Tabla 5) (Whiting, 1990; Kilby, 1999; Vallone, 2002; Romero y col., 2005; Palma, 2006). Lo anterior pudo ser motivado por el manejo del riego a que ha sido sometida la plantación de los cultivares, en donde el mismo se ha manejado con niveles deficitarios de agua. Al momento de la colecta de las muestras en la etapa de floración, el suelo donde se encontraban los cultivares muestreados presentaba 160 centibares de tensión hídrica, índice que indica una muy baja disponibilidad de humedad. Algunos reportes exponen que la concentración de nutrientes puede ser afectada por varios factores ambientales y de manejo del cultivo, encontrándose dentro de ellos el nivel del riego como lo indica Shellie y Brown (2012), quienes reportan que el estrés hídrico induce cambios significativos en las concentraciones de los nutrientes dándose el caso de que la concentración de nitratos se incrementa de 2 a 12 veces cuando las parras son sometidas a un alto índice de estrés hídrico.

En el caso de la concentración de los elementos $\mathrm{Cu}^{++}$y $\mathrm{SO}_{4}$ ${ }^{2}$. los niveles se presentaron dentro de un rango de normal a bajo en base al nivel considerado como óptimo (Tabla 5), aun cuando se esperaba que estos elementos presentaran altas concentraciones, debido a que son muy abundantes en los suelos de la zona de estudio, situación que hace pensar que la vid tiene mecanismos de absorción que evitan una acumulación en exceso de estos elementos en sus tejidos para evitar niveles que puedan causar toxicidad.

\section{Concentración de nutrientes en la lámina de hojas durante floración}

Los datos de las concentraciones de nutrientes en este tipo de tejido, en esta etapa de desarrollo (Tabla 1), muestran que dichas concentraciones son normales o superiores a los estándares reportados como óptimos (Tabla 5), con excepción del $\mathrm{Mg}^{++}$que presentó niveles por debajo de los estándares normales, mientras que el $\mathrm{Zn}^{++}$presentó niveles muy superiores a lo normal (Vallone, 2002; Romero y col., 2005; Davenport y Horneck, 2011; Romero y col., 2013). La probable causa de la baja concentración de $\mathrm{Mg}^{++}$ registrada puede ser el bajo $\mathrm{pH}$ del suelo (4.5 a 5.5) donde están plantados los cultivares de vid, ya que con este nivel de $\mathrm{pH}$ se aumenta la concentración en el suelo de los cationes $\mathrm{Al}^{+++}$y $\mathrm{Fe}^{++}$, los cuales pueden inhibir la absorción de $\mathrm{Mg}^{++}$ (Upjohn y col., 2005). Así mismo, la concentración de la mayoría de los micronutrientes en este tipo de tejido estuvo dentro del rango normal, con excepción de la del boro que se presentó alta (Tablas 1 y 5) (Vallone, 2002; Romero y col., 2005; Davenport y Horneck, 2011). A pesar de ello, 
no se presentaron síntomas de toxicidad de boro en las plantas, los cuales se manifiestan como clorosis, necrosis marginal, reducción del tamaño de las hojas y acortamiento de entrenudos.

Tabla 1. Concentración de nutrientes en peciolos y lámina de hojas durante la floración en cultivares de vid para vino en la región serrana de Sonora (Cananea), México. 2014.

\begin{tabular}{lccc}
\hline Nutriente & $\begin{array}{c}\text { Concentración en etapa } \\
\text { de floración/tejido } \\
\text { peciolo }\end{array}$ & $\begin{array}{c}\text { lámina de hoja } \\
\text { Nivel de signifi- } \\
\text { cancia }(\mathbf{T} \leq \mathbf{t}) \\
(\mathbf{T}<\mathbf{0 . 0 1})\end{array}$ \\
\hline $\mathrm{NO}_{3}^{-1} \mathrm{ppm}$ & 3034 & 1537 & $* *$ \\
\hline $\mathrm{HPO}_{4}^{-2}$ ppm & 5127 & 3245 & $* *$ \\
\hline $\mathrm{K}^{+} \%$ & 3.24 & 1.67 & $* *$ \\
\hline $\mathrm{Ca}^{++} \%$ & 2.48 & 2.04 & $* *$ \\
\hline $\mathrm{Mg}^{++} \%$ & 0.32 & 0.19 & $* *$ \\
\hline $\mathrm{Zn}^{++} \mathrm{ppm}$ & 41 & 32 & $* *$ \\
\hline $\mathrm{BO}_{4} \mathrm{ppm}$ & 37 & 43 & $* *$ \\
\hline $\mathrm{Fe}^{++} \mathrm{ppm}$ & 83 & 139 & $* *$ \\
\hline $\mathrm{Mn}^{++} \mathrm{ppm}$ & 132 & 145 & $* *$ \\
\hline $\mathrm{Na}^{+} \%$ & 0.19 & 0.16 & $\mathrm{NS}$ \\
\hline $\mathrm{Cu}^{++} \mathrm{ppm}$ & 10.03 & 10.68 & $\mathrm{NS}$ \\
\hline $\mathrm{SO}_{4}^{-2} \%$ & 0.174 & 0.17 & \\
\hline$*$
\end{tabular}

$* \mathrm{~T}<0.05 * * \mathrm{~T}<0.01$

El cálculo de los coeficientes de variación de las concentraciones de nutrientes en cada etapa del cultivo de la vid permite deducir la etapa más adecuada para realizar los muestreos para el análisis de los nutrimentos.

Con respecto al mejor tejido a utilizar para la toma de muestras para efectuar el análisis del contenido nutrimental de las plantas de vid, Robinson y col. (1978) proponen que, para determinar los elementos móviles como nitrógeno, fósforo, potasio y magnesio, se utilice de preferencia el peciolo, mientras que para elementos móviles como inmóviles se utilice la lámina de las hojas. Los datos registrados en el presente estudio se presentan en la Tabla 2, donde se puede observar que ocho de los 12 nutrientes analizados presentaron unos coeficientes de variación menores en el peciolo, que en la lámina de las hojas, lo cual demuestra que el pecíolo es el mejor tejido para analizar la mayoría de los nutrimentos de interés agronómico en la vid. A pesar de ello, es importante indicar que para cada variedad y portainjerto puede existir diferente capacidad de absorción y translocación de nutrientes, por lo que es importante hacer estudios específicos para tales combinaciones (Reuters y Robinson, 1977; Robinson, 1999; Fallahi y col., 2005; Romero y col., 2013).
Tabla 2. Coeficientes de variación de concentración de nutrientes en peciolos y lámina de hojas durante la floración en cultivares de vid para vino la región serrana, de Sonora (Cananea), México.

\begin{tabular}{|lcc|}
\hline & \multicolumn{1}{c}{$\begin{array}{c}\text { Coeficientes de variación de } \\
\text { Concentración de nutrientes en floración }\end{array}$} \\
\hline Nutriente & peciolos* & lámina de hojas \\
\hline $\mathrm{NO}_{3}^{-1}$ ppm & 19.8 & 21 \\
\hline $\mathrm{HPO}_{4}^{-2}$ ppm & 16.3 & 27 \\
\hline $\mathrm{K}^{+} \%$ & 24.6 & 18.6 \\
\hline $\mathrm{Ca}^{++} \%$ & 11.5 & 7.2 \\
\hline $\mathrm{Mg}^{++} \%$ & 20.9 & 10.5 \\
\hline $\mathrm{Zn}^{++}$ppm & 23.5 & 29.9 \\
\hline $\mathrm{BO}_{4} \mathrm{ppm}$ & 2.9 & 3.8 \\
\hline $\mathrm{Fe}^{++}$ppm & 20.8 & 30.9 \\
\hline $\mathrm{Mn}^{++}$ppm & 39.2 & 16.2 \\
\hline $\mathrm{Na}^{+} \%$ & 14.1 & 22.4 \\
\hline $\mathrm{Cu}^{++} \mathrm{ppm}$ & 10.1 & 17.9 \\
\hline $\mathrm{SO}_{4}^{-2} \%$ & 8.3 & 13.7 \\
\hline$* \mathrm{~N}=29$ & & \\
\hline
\end{tabular}

\section{Concentración de nutrientes en peciolos durante floración y envero}

Los datos de las concentraciones de macro y micronutrientes registrados en tejido de peciolos, durante la etapa de floración y envero en los cultivares considerados, se presentan en la Tabla 3. Estos datos muestran que la concentración de los elementos $\mathrm{HPO}_{4}^{-2}, \mathrm{BO}_{4}^{-}, \mathrm{Cu}^{++}$, y $\mathrm{SO}_{4}^{-2}$ ocurrieron dentro del rango de suficiencia, mientras los otros elementos presentaron niveles más altos que los considerados como óptimos a excepción del $\mathrm{Na}^{+}$que se presentó con niveles por debajo del que puede causar toxicidad (Tabla 5), (Kilby, 1999; Romero y col., 2005; Palma, 2006); así mismo ninguno de ellos presentó niveles que puedan inducir síntomas de toxicidad, lo cual se manifiesta con reducción del crecimiento de la parra y cambio en la coloración de las hojas.

Al efectuar la comparación de la concentración de elementos por etapas de desarrollo de la planta, la concentración de nitratos se reporta significativamente menor $(p<0.01)$, en alrededor de $50 \%$ en la etapa de envero, en relación a la etapa de floración (Tabla 3). Estos resultados concuerdan con lo reportado por Dow y col., (1983) y Ruiz (2000). Así mismo, las concentraciones de los elementos $\mathrm{HPO}_{4}^{-2}, \mathrm{Cu}^{++}$ y $\mathrm{SO}_{4}^{-2}$ fueron significativamente menores $(\mathrm{p}<0.01)$ durante el envero con respecto a floración, lo cual puede ser causa de que dichos nutrientes sean movilizados hacia los órganos demandantes dela planta, disminuyendosus concentraciones en los peciolos. Por otro lado, la concentración del resto de 
nutrientes fue mayor en la etapa de envero con respecto a la etapa de floración $(p<0.01)($ Tabla 3$)$, por lo que es posible que estos nutrientes no sean altamente demandados por los órganos en crecimiento o sean más inmóviles, lo que provoca su acumulación en los pecíolos. En forma general, los datos del análisis de los nutrimentos de los cultivares de vid para vino, efectuado en el presente estudio, permiten constatar que las concentraciones óptimas se reportan con más frecuencia en la fase de floración que en la fase de envero (Tabla 3).

Tabla 3. Concentración de nutrientes en peciolos, al momento de la floración y envero en cultivares de vid para vino, en la región serrana de Sonora (Cananea), México. 2014 y 2012.

\begin{tabular}{lccc} 
Nutriente & $\begin{array}{c}\text { Concentración de nutrientes en } \\
\text { peciolos/etapa }\end{array}$ & $\begin{array}{c}\text { Nivel de } \\
\text { significancia } \\
(\mathbf{T} \leq \mathbf{t}) \\
\mathrm{T}<\mathbf{0 . 0 1}\end{array}$ \\
\hline $\mathrm{NO}_{3}^{-1} \mathrm{ppm}$ & 3044 & 1810 & $* *$ \\
\hline $\mathrm{HPO}_{4}^{-2} \mathrm{ppm}$ & 5109 & 1565 & $* *$ \\
\hline $\mathrm{K}^{+} \%$ & 3.2 & 4.4 & $* *$ \\
\hline $\mathrm{Ca}^{++} \%$ & 2.5 & 3.1 & $* *$ \\
\hline $\mathrm{Mg}^{++} \%$ & 0.32 & 0.61 & $* *$ \\
\hline $\mathrm{Zn}^{++} \mathrm{ppm}$ & 41.0 & 74.3 & $* *$ \\
\hline $\mathrm{BO}_{4} \mathrm{ppm}$ & 37.7 & 38.2 & $* *$ \\
\hline $\mathrm{Fe}^{++} \mathrm{ppm}$ & 84.0 & 131.2 & $* *$ \\
\hline $\mathrm{Mn}^{++} \mathrm{ppm}$ & 134 & 408 & $* *$ \\
\hline $\mathrm{Na}^{+} \%$ & 0.19 & 0.37 & $* *$ \\
\hline $\mathrm{Cu}^{++} \mathrm{ppm}$ & 10.0 & 8.5 & $* *$ \\
\hline $\mathrm{SO}_{4}^{-2} \%$ & 0.17 & 0.10 & $* *$ \\
\hline
\end{tabular}

** $\mathbf{T}<0.01$

Los valores de coeficientes de variación de los elementos $\mathrm{NO}_{3}^{-1}, \mathrm{~K}^{+}, \mathrm{Mg}^{++}$y $\mathrm{BO}_{4}$, (Tabla 4) muestran que estos son menores en la etapa de envero que en floración, valores que indican que es mejor monitorear dichos nutrientes en la etapa de envero; en cambio para los elementos $\mathrm{HPO}_{4}^{-}$ ${ }^{2}, \mathrm{Ca}^{++}, \mathrm{Cu}^{++}$y $\mathrm{SO}_{4}{ }^{-2}$ es mejor hacer un monitoreo en la floración ya que presentan coeficientes de variación menores en dicha etapa.
Tabla 4. Coeficientes de variación de concentración de nutrientes en peciolos durante la floración y envero en cultivares de vid para vino en la región noreste de Sonora (Cananea), México. 2014.

\begin{tabular}{lcc} 
Concentración/ & $\begin{array}{c}\text { Coeficientes de variación de concentración } \\
\text { de nutrientes/ peciolos }\end{array}$ \\
nutrientes & Floración & Envero \\
\hline $\mathrm{NO}_{3}^{-1} \mathrm{ppm}$ & 19.8 & 14.0 \\
\hline $\mathrm{HPO}_{4}^{-2} \mathrm{ppm}$ & 16.3 & 27.0 \\
\hline $\mathrm{K}^{+} \%$ & 24.6 & 9.0 \\
\hline $\mathrm{Ca}^{++} \%$ & 11.5 & 16.0 \\
\hline $\mathrm{Mg}^{++} \%$ & 20.9 & 14.0 \\
\hline $\mathrm{Zn}^{++} \mathrm{ppm}$ & 23.5 & 24.0 \\
\hline $\mathrm{BO}_{4} \mathrm{ppm}$ & 2.9 & 2.0 \\
\hline $\mathrm{Fe}^{++} \mathrm{ppm}$ & 20.8 & 23.0 \\
\hline $\mathrm{Mn}^{++} \mathrm{ppm}$ & 39.2 & 34.0 \\
\hline $\mathrm{Na}^{+} \%$ & 14.1 & 27.0 \\
\hline $\mathrm{Cu}^{++} \mathrm{ppm}$ & 10.1 & 19.0 \\
\hline $\mathrm{SO}_{4}^{-2} \%$ & 8.3 & 100.0 \\
\hline
\end{tabular}

$* \mathrm{~N}=29$

Los datos de las concentraciones de los nutrimentos analizados (Tablas 1 y 3 ), así como las desviaciones estándar y coeficientes de variación (Tablas 2 y 4) de estos elementos en las etapas de desarrollo de las variedades de vid, dieron una idea de las variaciones que se presentaron en el análisis de macro y micro elementos en los diferentes cultivares muestreados. Los coeficientes de variación que son superiores al $20 \%$ indican que ocurrió una alta variación en la concentración de nutrientes entre los cultivares y los inferiores a ese porcentaje indican que existe una similitud en la concentración de los nutrientes entre los cultivares.

Por otro lado, los resultados obtenidos con respecto a las concentraciones de los diferentes nutrimentos analizados en el presente estudio muestran que las concentraciones de algunos de los nutrientes en la fase de envero son más altas que las esperadas, de acuerdo a los datos de la Tabla 5. Lo anterior posiblemente sea consecuencia de que las plantas muestreadas no tenían racimo y que por lo tanto los nutrientes no fueron utilizados y se acumularon en este tejido. Por otra parte, el crecimiento vegetativo también estuvo controlado mediante el riego, por lo que no hubo brotes con crecimiento excesivo que utilizaran los nutrientes y provocaran una disminución de la concentración de la floración al envero.

Con el propósito de tener una referencia de las concentraciones de valores óptimos para cada uno de los nutrimentos analizados, se presentan los valores de 
referencias óptimos en cultivares de vid para vino reportados por varios autores en diferentes regiones del mundo en la Tabla 5.

Tabla 5. Concentración de nutrientes óptima en vid para vino en diferentes regiones del mundo, de acuerdo a diferentes fuentes de información.

\begin{tabular}{|c|c|c|c|c|}
\hline \multirow[t]{3}{*}{ Nutriente } & & \multicolumn{3}{|c|}{ Concentración nutriente } \\
\hline & \multicolumn{2}{|c|}{ Floración } & \multicolumn{2}{|l|}{ Envero } \\
\hline & $\begin{array}{l}\text { Lámina } \\
\text { de hoja }\end{array}$ & Peciolo & Peciolo & \\
\hline $\mathrm{NO}_{3}^{-1}$ & \multirow{6}{*}{$\%$} & 3.1 & 1.10 & 0.46 \\
\hline $\mathrm{HPO}_{4}^{-2}$ & & 0.28 & 0.30 & 0.09 \\
\hline $\mathbf{K}^{+}$ & & 1.12 & 1.85 & 1.55 \\
\hline $\mathrm{Ca}^{++}$ & & 2.3 & 1.60 & 2.28 \\
\hline $\mathbf{M g}^{++}$ & & 0.33 & 0.60 & 0.91 \\
\hline $\mathrm{SO}_{4}^{-2}$ & & & 0.15 & $-\because-$ \\
\hline $\mathrm{NO}_{3}^{-1}$ & \multirow{7}{*}{ ppm } & \multicolumn{3}{|c|}{600} \\
\hline $\mathbf{Z n}^{++}$ & & 17 & 15 & 21.3 \\
\hline $\mathrm{Cu}^{++}$ & & 13 & 7.5 & 13 \\
\hline $\mathrm{Mn}^{++}$ & & 94 & 23 & 60 \\
\hline $\mathrm{Fe}^{++}$ & & 140 & 25 & 25 \\
\hline $\mathrm{BO}_{4}$ & & 36 & 40 & 50 \\
\hline $\mathrm{Na}^{+}$ & & & $<5000$ & 37 \\
\hline
\end{tabular}

Fuentes: Kilby, 1999; Vallone, 2002; Romero-I, 2005; Palma, 2006; Davenport y Horneck, 2011.

\section{CONCLUSIONES}

La concentración de los nutrientes $\mathrm{NO}_{3}^{-1}, \mathrm{HPO}_{4}^{-2}, \mathrm{~K}^{+}, \mathrm{Ca}^{++}$, $\mathrm{Mg}^{++}, \mathrm{Zn}^{++}, \mathrm{Na}^{+}$fue estadísticamente $(\mathrm{p}<0.05)$ mayor en pecíolo que en la hoja, en la época de floración. En contraste, el contenido de los elementos $\mathrm{BO}_{4^{\prime}} \mathrm{Fe}^{++}$y $\mathrm{Mn}^{++}$ fue mayor ( $p>0.05$ ) en hoja, mientras que en el contenido de $\mathrm{Cu}^{++}$y $\mathrm{SO}_{4}^{-2}$ no hubo diferencias $(\mathrm{p}>0.05)$ entre peciolo y hoja. El contraste entre las etapas fenológicas indica que la concentración de $\mathrm{NO}_{3}^{-1}, \mathrm{HPO}_{4}^{-2}, \mathrm{~K}^{++}, \mathrm{Mg}^{++}, \mathrm{Mn}^{++}, \mathrm{BO}_{4}^{-}$ en peciolos fue mayor $(p>0.05)$ durante la floración que durante el envero, mientras que en el resto de los elementos se registró lo contrario. Las notables diferencias, entre estos resultados y los consignados en la literatura como contenidos óptimos, hacen necesario continuar esta investigación para establecer índices de suficiencia nutrimental para la vid en condiciones de clima desértico.

\section{BIBLIOGRAFÍA}

Anónimo. 1996. Analytical methods for atomic absorption spectrometry. The Perkin Elmer Inc.

Christensen, P. 1984. Nutrient level comparisons of leaf petioles and blades in twenty-six grape cultivars over three years (through 1979 -1981). American Journal of Viticulture and Enology 35: 124-133.

Davenport, J.R., Marden, J.M., Mills, L.J. y Hattendorf, M.J. 2003. Response of Concord grape to variable rate nutrient management. American Journal of Viticulture and Enology 54 (4): 286-293.

Davenport, R. y Horneck, D.A. 2011. Sampling guide for nutrient assessment of irrigated vineyard. In Inland Pacific Northwest. Copyright. Washington State University: 1-6.

Dow, A.I., Clore, W.J., Halvorson A.R. y Tukey, R.B. 1983. Fertilizer Guide: Irrigated Vineyards. Washington State University Publication FG-13.

Fallahi, E., Shafii, B., Starck, J.C., Fallahi, B. y Hafez, S.L. 2005. Influence of wine grape cultivars on growth and leaf blade and petiole nutrients. Hortechology 15 (4): 825-830.

García, E. 1988. Modificaciones al sistema de clasificación climática de Köppen (para adaptarlos a las condiciones de la Republica Mexicana). 4ta. Ed. Offset Larios. Mexico D. F.

Huvely, H., Peto, J., Polos, E. y Cserni, I. 2014. Changes in nutrient content of grape leaves according to weather changes. Lucrari Stiinfice, Seria I. XV (2): 109-114.

Kilby, M. 1999. Wine grape nutritional survey. University of Arizona. College of Agriculture. Ag.arizona.edu/pubs/ crops/az1148/az1148-5pdf.

Márquez, J.A., Osorio, A., Martínez G., Núñez, J. H., Fú, A., Grageda, J., Valdez, B., Miranda, J.L. y Ávila, J. 2004. La vid, establecimiento y manejo en la Costa de Hermosillo y Pesqueira. Folleto Técnico Núm. 27. INIFAP-CIRNO$\mathrm{CECH}$, Hermosillo, Sonora.

Martínez, G., y Moreno, C.A. 2016. Vid para vino de mesa en el norte de Sonora, manejo de la relación fuente demanda. Presentado en: XIX Congreso Internacional de Ciencias Agrícolas. Mexicali. Baja California. Octubre 27-29.

Nagarajah, S. 1999. A petiole sap test for nitrate and potassium in Sultana grapevines. Australian Journal of Grape and Wine Research. 5: 56-60.

Palma, J. 2006. Guía de manejo nutrimental vegetal de especialidad uva de mesa. Manual Cropkyt SOM. Chile.

Peel, M.C., Finlayson, B.L. y McMahon, T.A. 2007. Updated world map of Koppen-Geiger climate classification. Hydrology and Earth System Sciences Discussions. 4: 439-473. 
Reuters, D.J. y Robinson, M.J. 1977. Plant analysis, an interpretation manual. 2nd. Ed. CSIRO Publishing.

Robinson, J.A., Nicholas P.R., y Carthy, Mc. 1978. A comparison of three methods of tissue analysis for assessing the nutrient status of plantings of Vitis vinifera in an irrigated area in South Australia. Journal of Experimental Agriculture and Animal Husbandry. 18: 294-300.

Robinson, T.B. 1999. Grape nutrition. In: B. G. Coombe and P. D. Dry (Eds.) Viticulture Vol 2. Practices. Hyde Park Press. Adelaide South Australia: 178-208.

Romero, I., Rueda, I.M., Iñigo, I.L., García, C., Villa, M.T., López, D., Ibáñez, P.S, Arroyo, M.C. y García, E.D. 2005. Diagnóstico nutricional de Vitis vinifera $\mathrm{L}$ Cv. Tempranillo en la D.O.C.A Rioja, niveles críticos preliminares. Zubia Monografico No. 16-17: 41-58.

Romero, I., García, E. y Martin, I. 2013. Leaf blade versus petiole analysis for nutritional diagnosis of Vitis vinifera L. cv Tempranillo. American Journal of Viticulture and Enology 64(1): 50-64.
Ruiz, S.R. 2000. Dinámica nutricional en cinco parrones de diferente productividad del valle central regado de Chile. Agricultura Técnica. 60: 379-398.

Shellie K. y Brown, B. 2012. Influence of deficit irrigation on nutrient indices in wine grape (Vitis vinifera L.). Agricultural Sciences 3(2): 268-273.

Upjohn, B., Fenton, G. y Conyers, M. 2005. Soil acidity and liming. NSW Department of Primary Industries. Australia. Agfact. A.C. 19.

Vallone, R. 2002. Nutrición y fertilización en vid. FCA-INTAMendoza. Argentina.

Whiting, J. 1990. Survey of petiole nutrient levels in grapevines grafted to rootstock in north-eastern and Southern Victoria. Report. Grape and Wine Research and Development Corporation. Victoria Australia.

Yaalon, D.H. 1997. Soils in the Mediterranean region: What makes them different? Catena 28: 157-169. 